\title{
Erratum to: One amino acid substitution in phytoene desaturase makes Chlorella zofingiensis resistant to norflurazon and enhances the biosynthesis of astaxanthin
}

Jin Liu $\cdot$ Yujuan Zhong $\cdot$ Zheng Sun · Junchao Huang • Yue Jiang $\cdot$ Gerhard Sandmann $\cdot$ Feng Chen

Published online: 4 April 2010

(C) Springer-Verlag 2010

\section{Erratum to: Planta}

DOI 10.1007/s00425-010-1132-y

Unfortunately, one of the co-author names (Yue Jiang) was missed in the published online first article. The correct author group should be

Jin Liu, Yujuan Zhong, Zheng Sun, Junchao Huang, Yue Jiang, Gerhard Sandmann, Feng Chen

Dr. Yue Jiang's address is: Department of Biology, Hong Kong Baptist University, Kowloon Tong, Hong Kong, China.

The online version of the original article can be found under doi:10.1007/s00425-010-1132-y.

J. Liu $\cdot$ Y. Zhong $\cdot$ Z. Sun $\cdot$ J. Huang $(\bowtie) \cdot$ F. Chen $(\bowtie)$

School of Biological Science,

The University of Hong Kong, Pokfulam Road,

Hong Kong, China

e-mail: huangjc@hku.hk

F. Chen

e-mail: sfchen@hku.hk

Y. Jiang

Department of Biology, Hong Kong Baptist University,

Kowloon Tong, Hong Kong, China

G. Sandmann

Bioscience, Goethe University Frankfurt,

P.O. Box 111932, 60054 Frankfurt, Germany 